\title{
Variability analysis of urban traffic noise measurands
}

\author{
Andrzej Bąkowski ${ }^{1,{ }^{*}}$, Leszek Radziszewski ${ }^{1}$, Pawet Świetlik ${ }^{2}$, and Vladimir Dekýš ${ }^{3}$ \\ ${ }^{1}$ Kielce University of Technology, Faculty of Mechatronics and Mechanical Engineering, Aleja \\ Tysiąclecia Państwa Polskiego 7, 25314 Kielce Poland \\ ${ }^{2}$ Kielce University of Technology, Faculty of Environmental, Geomatic and Energy Engineering, \\ Aleja Tysiąclecia Państwa Polskiego 7, 25314 Kielce Poland \\ ${ }^{3}$ University of Žilina, Univerzitná 8215/1, 01026 Žilina, Slovakia
}

\begin{abstract}
The paper presents the analysis results of measurements recorded by road traffic and noise monitoring station. The station is located in a medium size town in Poland (Kielce) situated at the national road to Cracow. The traffic flow was measured over the period between January and December 2013 for twenty four hours a day. Statistical analysis methods were used to determine the variability and uncertainty of the results. The measurements from two vehicular lanes running towards the town and two lanes running towards Cracow were analyzed. The variability of the results was described using parameters such as the coefficient of variation and positional variation. The results of vehicle traffic measurements were used to simulate changes in the noise measurand variation within 1-hour intervals. The Nordic Prediction Method was used for this purpose. It was found that in most cases, the distribution of the tested variable was not normal. Box plots were used to assess whether outliers data occurred in the recorded results. The variability of noise and type A uncertainty was evaluated.
\end{abstract}

Keywords: traffic noise, vehicles flow, predictive models

\section{Introduction}

In accordance with the applicable standards, measurements and calculations of road traffic noise measurands are performed for the overall period of 24 hours and for three time intervals within this period (day, evening, night), whereas the road vehicle traffic measurands are recorded every 1 hour. Commonly used noise measurand - the equivalent sound level - is an average value, therefore it does not accurately reflect changes in the values of noise parameters, which also have an impact on the assessment of its nuisance. One solution to this problem is to use the model of road traffic noise and to determine its measurands in more intervals of the 24-h period. In this paper, the calculations of noise measurands were performer every 1 hour, which resulted from the technical parameters of the traffic movement measuring terminal. Vehicle noise models fall into empirical models, such as the Nordic

\footnotetext{
* Corresponding author: abakowski@tu.kielce.pl

Reviewers: Milan Vaško, Zbigniew Kłos
} 
Prediction Method (NPM) and CoRTN, analytical models, such as Nord 2000 and CnossosEU, and stochastic models [1]. The NPM model [2] was chosen for this study due to its versatility and agreement of the measurement data obtained with the requirements of the model.

\section{Monitoring stations - traffic volume and noise measurements}

Traffic volumes analyzed in this study were measured by the permanent station recording traffic volume and sound pressure levels, located in Krakowska Street in Kielce [3]. The fourlane Krakowska Street is the main part of the outward route from the centre of Kielce towards Kraków, and carries both urban and transit traffic. The traffic monitoring station is located between two intersections at a distance of about $500 \mathrm{~m}$. The station includes a road radar box, a sound level meter and a weather station. The traffic volume was measured by WAVETRONIX digital radar with an operating frequency of $245 \mathrm{MHz}$. The acoustic measurements were carried out with the SVAN 958A a class 1 sound level meter. The Microtech Gefell MK250 free-field, prepolarized 1/2" condenser microphone with a sensitivity of $50 \mathrm{mV} / \mathrm{Pa}$ and a SV $12 \mathrm{~L}$ preamplifier was used. The microphone for the sound pressure measurements was mounted at a distance of $4 \mathrm{~m}$ from the edge of the road at a height of $4 \mathrm{~m}$. The weather data were recorded by means of a VAISALA WTX 510 automatic weather station.

The measurements were carried out for 24 hours a day throughout the year 2013. The traffic volume and speed data were recorded every 1 minute (buffer) and the averaged results were reported every 1 hour. The counts were used to calculate the traffic volume (understood as the sum of the number of passenger vehicles, delivery vans, lorries and single-track vehicles recorded within a time interval at all four lanes) and speed, split into seven days of the week, hours and three sub-intervals of a 24-hour interval: day, evening, and night. The results of acoustic measurements were also split into three sub-intervals of a 24-hour interval. The $R M S$ values of the $A$-weighted sound pressure were registered in the buffer every $1 \mathrm{~s}$ and the results were recorded every 1 minute. The results analyzed were two sets of data, the first containing the results of vehicle traffic measurements (split into groups of vehicles) together with vehicle average speeds for four road lanes, and the second containing the results of the equivalent sound level measurements recorded for three time intervals. Analysis was based on the measurements from only those days on which the terminal recorded correct data during the whole 24-hour measurement period (three results of the measurement of the equivalent sound level and 24 records containing traffic measurements and vehicle average speeds). The final size of the data under analysis was 903 records for the sound level (301 full measurement days) and 8208 records with vehicle traffic results (342 full measurement days). The set of data (acoustic measurements and vehicle traffic) included 296 days the measurement results of which formed the basis for further analysis.

\section{Noise measurands under analysis}

The most common noise indicator used to assess annoyance is the equivalent sound level $\left(L_{A e q, T}\right)$, expressed in $(\mathrm{dB})$, defined as [4]:

$$
\mathrm{L}_{\text {Aeq, } \mathrm{T}}=10 \cdot \log \left[\frac{1}{\mathrm{~T}} \int_{0}^{\mathrm{T}}\left(\frac{\mathrm{p}_{\mathrm{A}}(\mathrm{t})}{\mathrm{p}_{0}}\right)^{2} \mathrm{dt}\right]=10 \cdot \log \left[\left(\frac{\mathrm{p}_{\mathrm{A}_{\mathrm{RMS}}}}{\mathrm{p}_{0}}\right)^{2}\right]
$$

where:

$T$ - represents the overall measurement time, $\mathrm{s}$

$p_{A}(t)-A$-weighted sound pressure level, $\mathrm{Pa}$ 
$p_{0}-$ is the standardized reference sound pressure of $20 \mu \mathrm{Pa}$

$p_{A_{R M S}}$ - represents the effective sound pressure.

Noise is assessed with the use of parameters such as $\mathrm{Q}_{10}, \mathrm{Q}_{50}, \mathrm{Q}_{90}$, where:

- the percentile $\mathrm{Q}_{10}$, defined as sound pressure level exceeded by the signal in $10 \%$ of the measurement period (av. peak level),

- the percentile $\mathrm{Q}_{50}$, defined as the sound pressure level exceeded by the signal in $50 \%$ of the measurement period (median),

- the percentile $\mathrm{Q}_{90}$, defined as sound pressure level exceeded by the signal in $90 \%$ of the measurement period (av. background noise level).

The non-linear character of logarithmic function changes is a limitation to the use of the $L_{\text {Aeq,T }}$ parameter. This impedes both the determination of standard deviation or measurement uncertainty and the performance of a comparative analysis. It may also affect the results of statistical tests for normal distribution of the data. Therefore, the authors of this paper decided to determine the $R M S$ sound pressure $\left(p_{A_{R M S}}\right)$ in the $T$ period from equation (1) and use this parameter in further analysis.

$$
\mathrm{p}_{\mathrm{A}_{\mathrm{RMS}}}=\mathrm{p}_{\mathrm{A}}=\sqrt{10^{\left(0.1 \cdot \mathrm{L}_{\mathrm{Aeq}, \mathrm{T}}\right)} \cdot \mathrm{p}_{0}^{2}}
$$

Standard uncertainty of the parameter, determined in the Type A evaluation, can be calculated from the following relationship [5]:

$$
\mathrm{u}_{\mathrm{A}}=\sqrt{\frac{1}{\mathrm{n}(\mathrm{n}-1)} \sum_{\mathrm{i}=1}^{\mathrm{n}}\left(\mathrm{p}_{\mathrm{A}_{\mathrm{RMS}}}-\overline{\mathrm{p}_{\mathrm{A}_{\mathrm{RMS}}}}\right)^{2}}
$$

In this study, the authors analyzed $p_{A_{R M S}}$ from (2) expressed in terms of $\mathrm{Pa}$ to be able to easily compare the fixed components (the predicted value and the median) and variable components (standard deviation, coefficient of variation - classical and positional) of the sound pressure signals recorded. The tests for the variable components contained in the signals were based on the classical and positional measures: standard deviation $\left(\sigma_{p_{A_{R M S}}}\right)$, coefficient of variation $(\mathrm{COV})$, quartile deviation $Q_{31}$, quartile variation coefficient $\left(V_{Q_{31}}\right)$. Standard deviation is an absolute measure commonly used for the analysis of sound pressure variable component. Standard deviation is estimated from (4), where $n$ is the amount of data:

$$
\sigma_{\mathrm{p}_{\mathrm{A}_{\mathrm{R} M S}}}=\sqrt{\frac{1}{\mathrm{n}-1} \sum_{\mathrm{i}=1}^{\mathrm{i}=\mathrm{n}}\left(\mathrm{p}_{\mathrm{A}_{\mathrm{RMS}}}-\overline{\mathrm{p}_{\mathrm{A}_{\mathrm{RMS}}}}\right)^{2}}
$$

This parameter defines the average variation in individual sound pressure values from the arithmetic mean. Standard deviation can be related to the expected value of the signal being analyzed to obtain the coefficient of variation $(\mathrm{COV})$. The $\mathrm{COV}$ is a dimensionless relative measure that can be used to directly compare the variable components in its several realizations. For the sound pressure tested, the $C O V$ can be expressed as [6]:

$$
\mathrm{COV}_{\mathrm{p}_{\mathrm{A}_{\mathrm{RMS}}}}=\mathrm{COV}=\frac{\sigma_{\mathrm{p}_{\mathrm{A}_{\mathrm{RMS}}}}}{\overline{\mathrm{p}_{\mathrm{A}_{\mathrm{RMS}}}}} \cdot 100 \%
$$

The value of the $C O V$ is greatly influenced by atypical data taken into account in the analyses. This influence is less significant when positional measures are used. The measure of dispersion of the variable is the average quartile deviation:

$$
\mathrm{Q}_{31}=0.5 \cdot\left[\mathrm{Q}_{3}\left(\mathrm{p}_{\mathrm{A}_{\mathrm{RMS}}}\right)-\mathrm{Q}_{1}\left(\mathrm{p}_{\mathrm{A}_{\mathrm{RMS}}}\right)\right]
$$


Quartile deviation is an absolute measure that defines the average variance of half of the measurement data around the median (after rejecting 25\% data with the lowest values and $25 \%$ data of the highest values of sound pressure), expressed in terms of Pascals. By relating it to the median, the positional coefficient of variation is calculated from (7):

$$
\mathrm{V}_{\mathrm{Q}_{31}}=\frac{\mathrm{Q}_{31}}{\mathrm{Med}} \cdot 100 \%
$$

The positional coefficient of variation is a positional measure of the data between the first and third quartiles. Thus, atypical data exert less influence on these coefficients. It has to be noted, however, that the data under analysis represent the measurements collected within 24hour periods, thereby atypical data cannot be regarded as erroneous measurements.

The primary aim of this study is analyzing acoustic pressure variability due to traffic and measurement uncertainty. The preliminary analysis of $\mathrm{L}_{\mathrm{eq}, \mathrm{T}}$ measurement results for the three sub-intervals of the 24-hour interval indicated insufficient amount of test data. Therefore in addition to using available road noise models, computer simulations for determining the equivalent sound level for each hour of the 24-hour interval were performed. The 2013 road traffic measurement data allowed applying the NPM [2] as a model. It is a statistical model often used in the literature worldwide. To make it easier for the reader to assess further results of this study, the main algorithms of the method used are shown below. The level of noise due to heavy vehicles can be calculated as follows:

$$
\begin{gathered}
\mathrm{L}_{\mathrm{AE} \text {,heavy }}=80.5+30 \log \left(\frac{\mathrm{v}}{50}\right) \text { for } 50 \leq \mathrm{v} \leq 90 \mathrm{~km} / \mathrm{h} \\
\mathrm{L}_{\mathrm{AE} \text {, heavy }}=80.5 \text { for } 30 \leq \mathrm{v}<50 \mathrm{~km} / \mathrm{h}
\end{gathered}
$$

The level of noise due to light vehicles can be calculated as follows:

$$
\begin{gathered}
\mathrm{L}_{\mathrm{AE}, \text { light }}=73.5+25 \log \left(\frac{\mathrm{v}}{50}\right) \text { for } 40 \mathrm{~km} / \mathrm{h} \leq \mathrm{v} \\
\mathrm{L}_{\mathrm{AE}, \text { light }}=71.1 \text { for } 30 \leq \mathrm{v}<40 \mathrm{~km} / \mathrm{h}
\end{gathered}
$$

The equivalent sound level for light or heavy vehicle groups and time $\mathrm{T}$ are as follows:

$$
\begin{aligned}
& \mathrm{L}_{\text {Aeq,T,light }}=\mathrm{L}_{\mathrm{AE}, \text { light }}+10 \log \left(\frac{\mathrm{N}_{\text {light }}}{\mathrm{T}}\right) \\
& \mathrm{L}_{\text {Aeq, T, heavy }}=\mathrm{L}_{\mathrm{AE}, \text { heavy }}+10 \log \left(\frac{\mathrm{N}_{\text {heavy }}}{\mathrm{T}}\right)
\end{aligned}
$$

$\mathrm{T}$ - the number of seconds within the time interval for which $L_{A e q, T}$ is calculated, $N_{\text {light }}, N_{\text {heavy }}$ - the number of light or heavy vehicles recorded at time T.

The equivalent sound level generated by both groups for $\mathrm{T}$ can be calculated from:

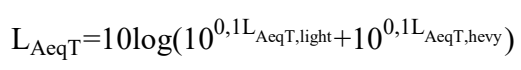

\section{Results}

Figure 1 compiles the measurement and calculated results for the equivalent sound level (for the day sub-interval ) expressed as $\mathrm{dB}$ for $T=43200 \mathrm{~s}$. The graphical representation of the spread of variables is shown on the box plots in Figs. 2 and 3. Figure 1 shows qualitative agreement between experimental and NPM calculated values. The discrepancies between these data can be estimated as follows: 


$$
\mathrm{RMSE}=\sqrt{\frac{1}{\mathrm{~N}} \sum_{\mathrm{i}=1}^{\mathrm{N}}\left(\mathrm{Leq}_{\mathrm{E}}-\mathrm{Leq}_{\mathrm{M}}\right)^{2}}
$$
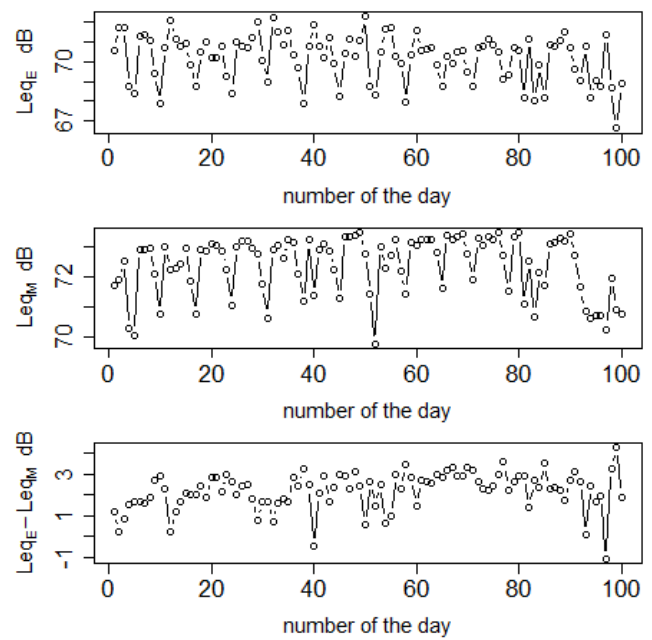

Fig. 1. The equivalent sound level determined for the day sub-interval - first 100 measurement days: $L e q_{E}$ - measurement data, $L e q_{M}$ - the values determined by the model, $L e q_{E}-L e q_{M}$ - the difference between the measurements and calculations

The value of this parameter varies within the 24-hour period: $\mathrm{RMSE}=2.45 \mathrm{~dB}$ for the day sub-interval, $\mathrm{RMSE}=2.34 \mathrm{~dB}$ for the evening sub-interval, and RMSE $=2.75 \mathrm{~dB}$ for the night sub-interval.

Figure $2 \mathrm{a}$ shows that from 3.00 to 5.00 the number of vehicles changes only slightly, whereas the noise level increases by approximately $3 \mathrm{~dB}$. Between 8.00 and 18.00, the number of vehicles changes noticeably (by around 1000) at the noise level remaining at about $73 \mathrm{~dB}$. Figure $3 \mathrm{~b}$ indicates that at weekends, the vehicle number decreases by about 1000 but the noise decreases by only $1 \mathrm{~dB}$.

a)
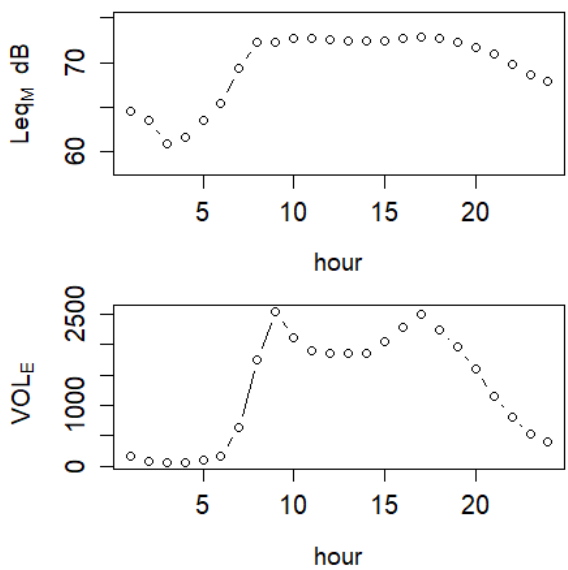

b)
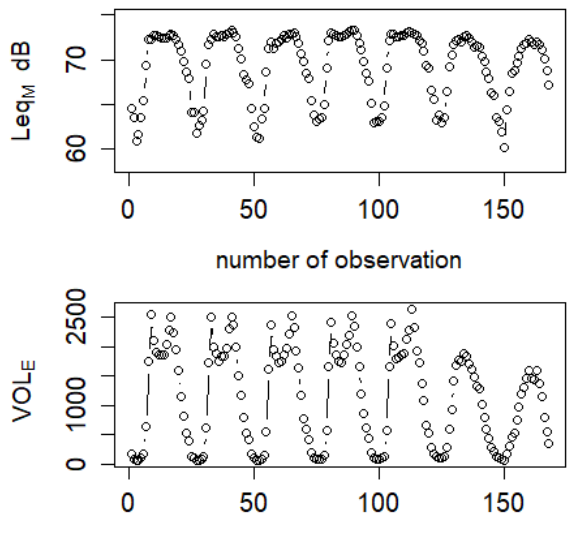

number of observation

Fig. 2. The equivalent sound level $L e q_{M}$ determined for one hour intervals and the corresponding numbers of recorded vehicles $V O L_{E}-$ a) arbitrarily chosen day (11.03.2013 Monday), b) arbitrarily chosen week from 11.03 to 17.03 .2013 
Table 1 summarizes the results of the $R M S$ sound pressure values calculated according to relationship (2) for three sub-intervals within the period of 24 hours.

Table 1. Statistical measures for sound pressure expressed in $\mathrm{mPa}$, corresponding to the equivalent sound levels for different sub-intervals of a 24 -hour period

\begin{tabular}{|l|c|c|c|c|c|c|c|c|c|}
\hline Data & $\begin{array}{c}\mathrm{Q}_{90} \\
\mathrm{mPa}\end{array}$ & $\begin{array}{c}\text { Med. } \\
\mathrm{mPa}\end{array}$ & $\begin{array}{c}\mathrm{Q}_{10} \\
\mathrm{mPa}\end{array}$ & $\begin{array}{c}\overline{\mathrm{p}_{\mathrm{R}_{\mathrm{RS}}}} \\
\mathrm{mPa}\end{array}$ & \begin{tabular}{c}
$\sigma_{\mathrm{p}_{\mathrm{A}_{\mathrm{RS}}}} \mathrm{mPa}$ \\
\multicolumn{8}{c|}{}
\end{tabular} & $\begin{array}{c}\mathrm{COV} \\
\%\end{array}$ & $\begin{array}{c}Q_{31} \\
\mathrm{mPa}\end{array}$ & $\begin{array}{c}\mathrm{Vq} \\
\%\end{array}$ & $\begin{array}{c}\mathrm{u}_{\mathrm{a}} \\
\mathrm{mPa}\end{array}$ \\
\hline DAY SUB-INTERVAL \\
\hline Measurement & 53.99 & 66.53 & 75.25 & 65.39 & 8.22 & 12.58 & 4.91 & 7.38 & 0.48 \\
\hline Model & 73.15 & 87.82 & 91.56 & 84.88 & 7.21 & 8.49 & 4.40 & 5.01 & 0.42 \\
\hline \multicolumn{8}{|c|}{ EVENING SUB-INTERVAL } \\
\hline Measurement & 49.32 & 55.02 & 63.61 & 55.79 & 6.02 & 10.79 & 3.72 & 6.75 & 0.35 \\
\hline Model & 65.11 & 71.46 & 76.74 & 71.15 & 4.90 & 6.89 & 2.97 & 4.16 & 0.28 \\
\hline \multicolumn{8}{|c|}{ NIGHT SUB-INTERVAL } \\
\hline Measurement & 30.12 & 34.68 & 38.90 & 34.57 & 3.64 & 10.52 & 2.02 & 5.83 & 0.21 \\
\hline Model & 39.22 & 45.77 & 57.21 & 46.55 & 6.60 & 14.17 & 3.55 & 7.75 & 0.38 \\
\hline
\end{tabular}

For the 24-hour period, the value of $\mathrm{Q}_{90}$, considered as the average background noise level [7], was about $32 \mathrm{mPa}$, whereas the value of $\mathrm{Q}_{10}$, considered as the average peak level, was about $87 \mathrm{mPa}$. The differences between the median values determined for the measurement and the NPM model data are about 30\%. Statistical analysis showed that the value of $Q_{31}$ calculated according to the NPM model was about $20 \mathrm{mPa}$ for the 24-hour period and was about $4 \mathrm{mPa}$ for the remaining sub-intervals. The value of $\mathrm{Vq}$ for the 24 -hour period was about $28 \%$ and ranged from $4 \%$ to $8 \%$ for the other sub-intervals. The difference in value between this parameter determined from the measurements and that from the NPM model ranged between $-2 \%$ and $3 \%$. The difference in value between $u_{\mathrm{A}}$ determined from the measurements and $\mathrm{u}_{\mathrm{A}}$ from the NPM is less than $0.2 \mathrm{mPa}$.

The results of Shapiro-Wilk and Jarque-Bera tests allow rejecting the null hypothesis about the normal distribution of the calculated data $\mathrm{p}_{\mathrm{A}_{\mathrm{RMS}}}$ at particular hours of the 24-hour period because the calculated limit significance levels, p-values, are lower than 0.05 - the value adopted as the required level of significance. But no evidence was found to reject the null hypothesis for hours 2.00, 6.00, 22.00, and 23.00.

Figure 3 shows parameters $\mathrm{Q}_{10}$ and $\mathrm{Q}_{90}$ and box diagrams for the sound pressure (calculated according to the NPM) at particular hours of the 24-hour period. Figure 3a shows that the gap between $\mathrm{Q}_{90}$ and $\mathrm{Q}_{10}$ varies across hours and increases at 4.00 to 6.00. The $\mathrm{Q}_{90}$ value increases till 15.00 and $Q_{10}$ increases until 9.00 to start decreasing at 16.00 . Figure $3 b$ shows a lot of outliers that were taken into account in the statistics. The outliers with values above the median for 6.00 occurred above all at 5.00.

Statistical analysis of the sound pressure values calculated according to the NPM for particular hours shows that:

- the values of $Q_{31}$ fall within the range $2.8 \mathrm{mPa}$ to $9.8 \mathrm{mPa}$,

- the values of $Q_{90}$ fall within the range $22 \mathrm{mPa}$ to $79 \mathrm{mPa}$,

- the values of $\mathrm{Q}_{10}$ fall within the range $37 \mathrm{mPa}$ to $92 \mathrm{mPa}$

- the values of $\mathrm{u}_{\mathrm{A}}$ fall within the range $0.26 \mathrm{mPa}$ to $0.82 \mathrm{mPa}$ at 7.00 hours,

- the values of $\mathrm{Vq}$ fall within the range $3 \%$ to $14 \%$. 
a)

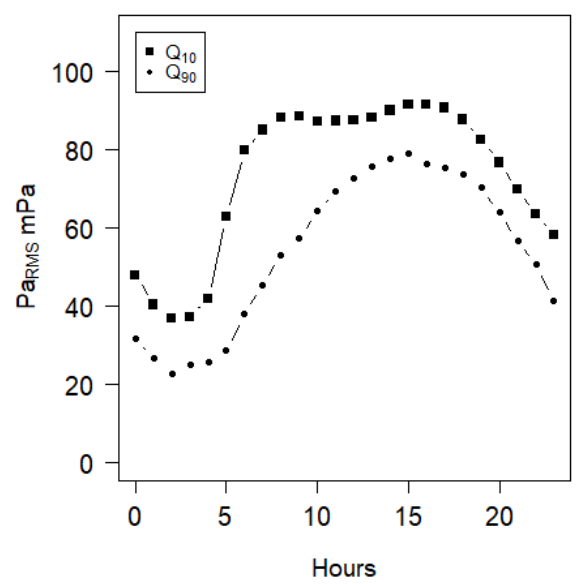

b)

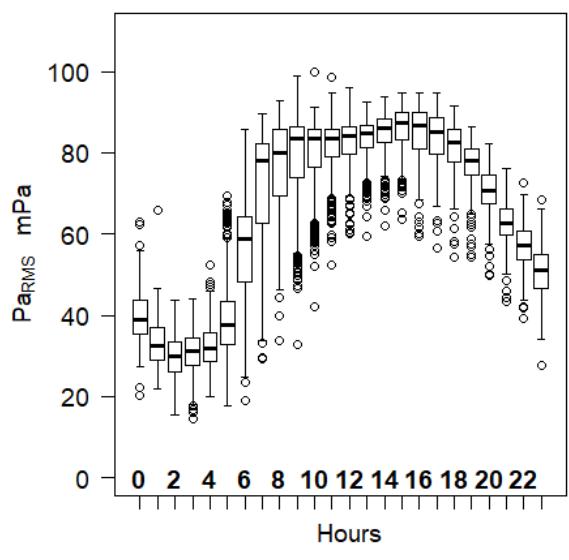

Fig. 3. Changes in sound pressure values (according to the NPM) at particular hours of the 24-hour period a) parameters $\mathrm{Q}_{10}$ and $\mathrm{Q}_{90}, \mathrm{~b}$ ) box plot of sound pressure diagrams

a)

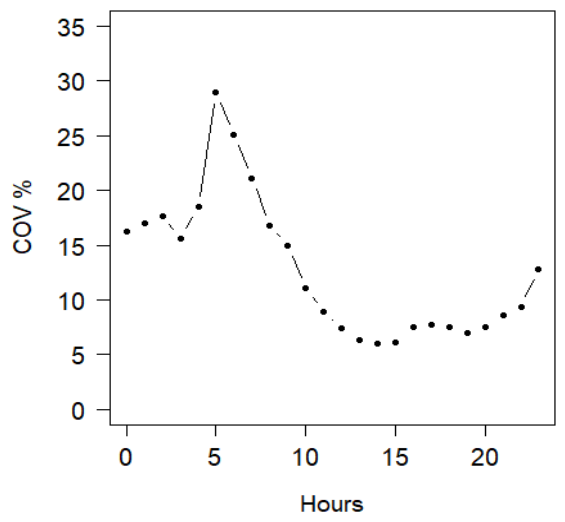

b)

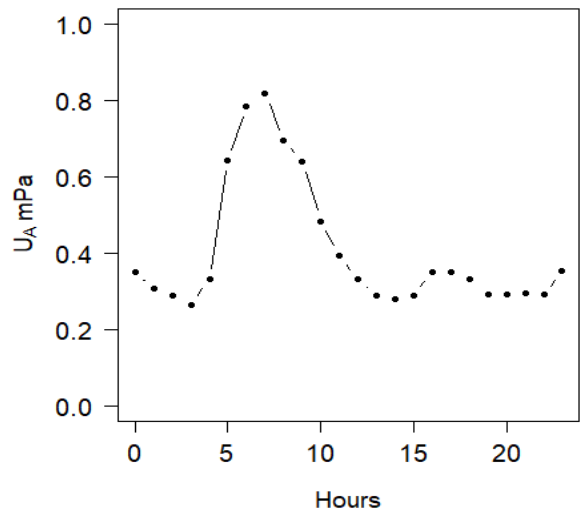

Fig. 4. Changes in the values of a) sound pressure coefficient of variation b) sound pressure standard uncertainty - (pressure calculated according to the NPM) at particular hours of the 24-hour period

Figure 4 shows changes in the values of COV and type A uncertainty. The COV is the highest at $5.00(29 \%)$ and decreases until $14.00(6 \%)$. The value of uncertainty $u_{a}$ is maximum at 7.00 and minimum at 14.00 .

\section{Discussion}

Analysis of Fig. 1 reveals some differences between the measured and calculated values of $L_{\text {Aeq }}$. They result from discrepancies between actual and model conditions of measurement, e.g., the measuring microphone was at the height of $4 \mathrm{~m}$ and distance of $4 \mathrm{~m}$ from the edge of the roadway, while according to the model, the microphone should be at a distance of $10 \mathrm{~m}$ from the source of noise at the height of $1.5 \mathrm{~m}$. In fact, each lane is the source of noise, thus there were four acoustic sources along the road section under analysis. Figure $3 \mathrm{~b}$ shows a large number of outliers, in particular those with low values, which can also cause the RMSE parameter to range from $2.34 \mathrm{~dB}$ to $2.75 \mathrm{~dB}$, depending on the time of the 24-hour interval. 
Despite some discrepancies with respect to experimental data, the applied noise model (NPM) allowed demonstrating that the average background noise level, COV and acoustic pressure measurement uncertainty vary from time sub-interval to sub-interval.

Analysis of Figures 2a, 3 and 4 indicates that optimum conditions for noise measurement occur from 13.00 to 15.00 . During these hours noise values change only slightly and the measurement uncertainty is the lowest.

\section{References}

1. N. Garg, S. Maji, EIA Review 46, 68-81 (2014)

2. H. Bendtsen, Sci Total Environ. 235, 331-338 (1999)

3. A. Bąkowski, L. Radziszewski, Z. Skrobacki, Procedia Engineering 177, 281-288 (2017)

4. J. Forssen, M. Horniks, Acta Acust united Ac, 92(6), 998-1008 (2006)

5. W. Batko, B. Stępień, ARCH. ACOUST. 39(1), 25-36 (2014)

6. A. Bąkowski, L. Radziszewski, M. Žmindak, Procedia Engineering 177 (2017)

7. J. Quartieri, N. E. Mastorakis, G. Iannone, C. Guarnaccia,S. D'Ambrosio, A. Troisi, TLL Lenza, Recent Advances in Applied and Theoretical Mechanics (2009) 